\title{
Identidad profesional, regulación y práctica de la Sociología y la Ciencia Política en España
}

\author{
Ángel Alonso Domínguez ${ }^{1}$ \\ Jacobo Blanco Fernández ${ }^{2}$ \\ Carlos Gil de Gómez Pérez-Aradros ${ }^{3}$
}

Fecha de recepción: 31 de julio de 2019

Fecha de aprobación: 27 de septiembre de 2019

Fecha de publicación: 31 de diciembre de 2019

\begin{abstract}
Resumen
El artículo explora el ejercicio profesional de la sociología y de la ciencia política en España, tomando en consideración el proceso de descentralización de sus colegios profesionales, la regulación normativa y las estrategias puestas en práctica para aumentar su presencia, influencia, poder, credibilidad y atractivo para los potenciales colegiados.

La investigación resulta novedosa puesto que el rol de las asociaciones profesionales como mecanismos reguladores ha sido ampliamente teorizado, pero es poco frecuente la utilización de metodología empírica para abordar la cuestión. El estudio se centra, en primer lugar, en la discusión estratégica sobre las profesiones; a continuación, se revisa la normativa y, finalmente, se explora el fenómeno a partir de las respuestas de un cuestionario dirigido a los representantes de las juntas de gobierno de los colegios territoriales.

Del análisis empírico se desprenden algunas conclusiones importantes sobre la inestabilidad e incertidumbre que afrontan la sociología y la ciencia política y sus dificultades para alcanzar un mayor desarrollo y penetración profesional. Las soluciones aportadas desde la perspectiva de los actores se encuentran muy polarizadas entre quienes abogan por el establecimiento de espacios interdisciplinares con campos profesionales próximos y los que consideran que es necesaria una corporatización fuerte.
\end{abstract}

Palabras clave: Ciencia política, colegios profesionales, ocupaciones, profesión, Sociología.

1 Doctor en Sociología por la Universidad de Oviedo. Departamento de Sociología. Universidad de Oviedo, Oviedo (España). Contacto: alonsodangel@uniovi.es

2 Doctor en Sociología por la Universidad Complutense. Pragma Sociología Aplicada, Oviedo (España). Contacto: jacoboblan@gmail.com

3 Licenciado en Ciencias Políticas y de la Administración por la Universidad Autónoma de Madrid. Gobierno del Principado de Asturias, Oviedo (España). Contacto: carlos.gildegomezperez-aradros@asturias.org 


\title{
Professional identity, regulation and practice of Sociology and Political Science in Spain
}

\begin{abstract}
The article explores the professional exercise of sociology and political science in Spain, taking into account the process of decentralization of its professional associations, its regulation, and the strategies put in place to increase its presence, influence, power, credibility and attractiveness to potential members. This research is novel since the role of professional associations as regulatory mechanisms has been widely theorized, but the use of empirical methodology to address the topic is less common. The study first focuses on the strategic discussion on the professions, followed by a review of the regulations, and, finally, the phenomenon is explored based on answers to a questionnaire addressed to the representatives of territorial associations.

The empirical analysis offers some important conclusions on the instability and uncertainty that sociology and political science face and their difficulties in achieving further development and professional penetration. The solutions provided from the perspective of the key players are highly polarized between those who advocate for the establishment of interdisciplinary spaces with related professional fields and those who believe that strong corporatism is necessary.
\end{abstract}

Key words: Political Science, profession, professional associations, occupations, sociology

\section{Identidade profissional, regulação e prática da Sociologia e das Ciências Políticas na Espanha}

\section{Resumo}

O artigo explora o exercício profissional da sociologia e das ciências políticas na Espanha, levando em consideração o processo de descentralização de seus conselhos profissionais, a regulação normativa e as estratégias aplicadas para aumentar sua presença, influência, poder, credibilidade e atrativo para os potenciais associados.

A investigação resulta inovadora uma vez que o papel das associações profissionais como mecanismos reguladores tem sido amplamente teorizado, mas é pouco frequente a utilização de metodologia empírica para abordar a questão. O estudo está centrado, em primeiro lugar, na discussão estratégica sobre as profissões; em seguida é revisada a normativa e, finalmente, o fenômeno é explorado a partir das respostas de um questionário dirigido aos representantes das diretorias dos conselhos territoriais.

Da análise empírica obtemos algumas conclusões importantes sobre a instabilidade e insegurança que enfrentam a sociologia e as ciências políticas e as dificuldades para alcançarem um maior desenvolvimento e penetração profissional. As soluções aportadas desde a perspectiva dos atores se encontram muito polarizadas entre os que defendem o estabelecimento de espaços interdisciplinares com campos profissionais próximos e os que consideram que é necessária uma corporatização forte.

Palavras-chave: Ciências Políticas, conselhos profissionais, ocupações, profissão, Sociologia. 


\section{Introducción ${ }^{4}$}

La discusión estratégica sobre la profesión sociológica y politológica ha sido tratada de manera profusa por la literatura científica desde hace décadas, pero el debate se ha mantenido intenso hasta nuestros días (Wilensky, 1964; Freidson, 1983; Abbott, 1988; Brint, 1990; Almond, 1999; Greenwood, Suddaby, \& Hinings, 2002; Bulcourf \& Vázquez, 2004; Fernández Alcalde, 2010; Blois, 2015; Fernández-Esquinas, 2016), bien sea desde un abordaje estático, en el que domina el análisis de las estructuras de las disciplinas, como dinámico, considerando el mayor desarrollo que han tenido algunas ocupaciones y las razones por las que han alcanzado una posición dominante sobre las demás. El conocimiento teórico-experto resulta clave para el anclaje de las profesiones y para la consecución de poder, presencia y difusión dentro de la sociedad (Freidson, 1983), a pesar de que, en ocasiones, parezca un tanto hermético e incluso esotérico como barrera para evitar el intrusismo profesional en áreas propias de su ámbito de conocimiento. Más allá de la importancia de trascender a la sociedad civil, Brint (1990) acercó el debate al intercambio de autonomía y poder que se establece entre las profesiones y otras organizaciones vitales para la supervivencia de la disciplina. Desde una perspectiva más amplia, ese trade-off define la correlación de fuerzas que las profesiones establecen cuando intentan delimitar su marco jurisdiccional (Abbott, 1988). Según este enfoque sistémico, las ocupaciones y las profesiones compiten por el dominio de determinados campos de actuación y por la obtención del prestigio profesional para nuevas áreas de conocimiento. El camino hacia la profesionalización es, por tanto, consecuencia de las disputas entre ocupaciones y profesiones, así como al interior

\footnotetext{
4 Nota respecto al lenguaje de género: conscientes de la importancia y necesidad de utilizar el lenguaje de género en el presente documento, pero con el fin de evitar la reiteración, se señala que el término "sociólogo/s" "politólogo/s" se refiere tanto a sociólogo/s como a socióloga/s y a politólogo/s y politóloga/s.
} 
de las distintas corrientes de las disciplinas (Wilensky, 1964; Abbott, 1988; Bourdieu, 2002).

Esta visión poliédrica va más allá del ejercicio profesional de la sociología y de la ciencia política, afectando tanto a la trascendente discusión sobre los límites de ambas disciplinas como de sus competencias y ámbitos de actuación. Por si no fuera suficiente, la controversia ha sido animada desde la Unión Europea, que ha abogado por la desregulación de los servicios profesionales y la descentralización de las competencias sobre colegios profesionales a las regiones, inmiscuyéndose de lleno en el debate sobre la creciente diversidad de titulaciones que proporcionan acceso a una misma profesión. Para rematar el escenario, no debe perderse de vista que la sociología y la ciencia política no han sido nunca actividades de colegiación obligatoria en España, lo que incrementa su vulnerabilidad y dificultad para competir con otras disciplinas afines.

A partir del planteamiento descrito, la estructura del artículo es la siguiente. En primer lugar, se aborda la discusión conceptual sobre el ejercicio de las profesiones. A continuación, se revisa el marco regulatorio de los colegios profesionales, así como el libre acceso y ejercicio de las actividades. En última instancia, el panorama se completa con la exploración del proceso de descentralización territorial a partir de las respuestas ofrecidas por representantes de las juntas de gobierno de los colegios profesionales de sociología y ciencia política. El análisis empírico nos ha permitido establecer algunas conclusiones y abrir un debate en torno a la inestabilidad e incertidumbre de ambas profesiones y sobre sus posibilidades de fortalecimiento.

\section{El ejercicio profesional de la Sociología: conflicto inter e intraprofesional}

Las asociaciones profesionales han mostrado su fortaleza como estructuras de intermediación al interior de las organizaciones al ser capaces de negociar y gestionar el debate sobre la 
identidad, reforzando la pertenencia, los vínculos intragrupales y otros aspectos menos perceptibles, tales como las convicciones, valores y creencias relacionadas con la profesión (Greenwood et al, 2002; Machuca, 2008; Barbour \& Lammers, 2015; Guilló \& Santiago, 2016), pero también se han mostrado como firmes baluartes a la hora de presentar la identidad fuera de la propia profesión. No obstante, para que una ocupación sea reconocida tanto por sus propios miembros como por la comunidad en la que está inserta, no es suficiente con que un grupo ocupacional controle los fundamentos epistemológicos de una determinada disciplina, ni tampoco que cuente con un procedimiento de instrucción y selección estructurado, sino que este proceso tiene que finalizar necesariamente en la constitución de una asociación profesional que le otorgue el reconocimiento público y el apoyo legal del ejercicio (Gómez-García, 2010). En este sentido, los colegios profesionales ejercen un papel fundamental para sus miembros, como proveedores de información especializada, generadores de perceptibilidad, beneficios de pertenencia y mejora del capital social, así como para establecer lo que Abbott (1988) denomina "jurisdicciones", es decir, puertas de acceso al poder y el prestigio que solamente se pueden alcanzar a través de la provisión y la vigilancia de "tareas, funciones, trabajos y tipos de recursos" (Fernández-Esquinas, 2016, p. 214).

No obstante, la sociología y la ciencia política, en España, constituyen profesiones no reguladas, no corporativas, y que, por tanto, carecen tanto de disposiciones jurídicas específicas como de una "jerarquía burocrática" (Bulcourf \& Vázquez, 2004, p. 291), con las repercusiones negativas que esto puede tener para el progreso de la profesión. Por ello, para ambas disciplinas resulta imprescindible una sólida cimentación de la identidad propia, que redunde en un elevado prestigio social y proyecte una imagen de mayor efectividad en el desempeño de determinadas tareas respecto a sus más inmediatas competidoras (Scott, 2008; Ashforth, Rogers \& Corley, 2011; Alonso-Domínguez, Eguren \& González Fernández, 2016; Fernández-Esquinas, 2016; Guilló \& 
Santiago, 2016). En el proceso mediante el cual las profesiones devinieron en ocupaciones, encontramos algunas de las claves para la consecución de autonomía, acceso al poder y establecimiento de sólidas jurisdicciones. Así, resulta notorio que han aumentado las rutas de acceso al ejercicio de algunas profesiones no reguladas, como es el caso de la sociología y la ciencia política, ocupaciones a las que se puede llegar desde disciplinas tan diversas como el trabajo social, la geografía o el marketing. En este caso, el poder que tengan los colegios profesionales para salvar sus jurisdicciones (en términos de influencia o afiliación) resulta crucial en la delimitación de sus espacios y campo profesional. Lamentablemente, como veremos más adelante en la sección dedicada a la implantación de la sociología y la ciencia política, los colegios profesionales de ambas disciplinas adolecen de baja afiliación y un exceso de fragmentación, con las implicaciones que esto conlleva para la acción colectiva, la generación de recursos y las limitaciones para la mejora del diseño organizacional (Fernández-Esquinas, 2016).

Resulta paradójico que la sociología, que tuvo un papel especialmente relevante en el análisis del proceso de etiquetaje y legitimación de las ocupaciones que conseguían alcanzar esa posición (Freidson, 1983; Nolin, 2008), se encuentre hoy en un momento de indefinición, desconexión con la práctica, e incluso alienación y disolución de la identidad profesional (Machuca, 2008; FernándezAlcalde, 2010; Blois, 2014; Alonso-Domínguez, et al., 2016; Guilló \& Santiago, 2016). Este hecho ha obligado a sus profesionales a la inserción laboral en ámbitos dominados por disciplinas cercanas (Fernández-Esquinas, 2016) que han ganado la disputa por el espacio social de acción y de influencia, así como en la competencia por el capital específico y la posición dominante en la distribución de poder (Bourdieu, 2002). Todas estas circunstancias han influido de manera decisiva en el incremento de la disonancia entre realidad y expectativas, y ha movido a los profesionales de la sociología a buscar reconocimiento social a través de la construcción de una "segunda identidad profesional" (Bolívar, Gallego, León 
\& Pérez, 2005, p. 26). De igual forma, la ciencia política, más allá de sus tradicionales dificultades para dilucidar su propia genealogía, o la división epistemológica típicamente definida como de "mesas separadas" (Almond, 1999), ha sufrido problemas de orden metodológico, derivados del enfoque jurídico de la disciplina, cuestiones que han lastrado su institucionalización, su desarrollo profesional y su evolución como campo científico al margen de la academia (Cotarelo, 1994; Bulcourf \& Vázquez, 2004).

El debilitamiento de la sociología como referente teórico en la discusión sobre la profesión es un síntoma de un proceso más profundo que afecta a la práctica de la disciplina y a la evolución de su campo profesional en España. Encontramos evidencias del debilitamiento de la sociología en el desencuentro entre las competencias aprendidas y las necesidades del mercado (Machuca, 2008; Fernández-Esquinas, 2016), las dificultades para mostrar las fortalezas de la profesión a los hipotéticos empleadores (Sigalat, 2016), la ausencia de corporativismo (Fernández Alcalde, 2010), la dilución del perfil profesional (Alonso-Domínguez et al., 2016), o las carencias en la institucionalización corporativa (Guilló \& Santiago, 2016). La marginalidad y la falta de reconocimiento de los colegios profesionales tampoco ha ayudado a la consolidación de la disciplina, un hecho que no es excepcional, sino que lo comparte con otros países que cuentan con una larga tradición sociológica pero con dificultades similares, tanto para buscar espacios de poder a través del prestigio profesional y la influencia social como para la generación de capital social que fomente el desarrollo de las organizaciones (Forni \& Nardone, 2007; Gildo de la Cruz, 2007; Machuca, 2008; Torini, 2012; Blois, 2015, 2017).

Tampoco la ciencia política ha sido capaz de seguir evolucionando en el desarrollo de la profesionalización de la disciplina, a diferencia de otras ciencias que hace tiempo que consiguieron mejorar su estatus a partir de la práctica. Del mismo modo que la sociología, la predominante orientación académica en España no parece haber resultado muy útil en la consolidación de la prácti- 
ca profesional, a pesar de que en los últimos tiempos parece haberse desprendido de esa dependencia. Así, podemos encontrar a la ciencia política cada vez más incardinada en la consultoría privada o en el asesoramiento de las administraciones públicas (AAPP), ámbitos en los que tradicionalmente ha sido difícil encontrar profesionales de la disciplina (Bulcourf \& Vázquez, 2004). También, al igual que la sociología, la anemia regulatoria y la vaga jurisdicción de sus asociaciones profesionales han resultado una rémora para su desarrollo como campo profesional, y el poder y el estatus que otorgan la exigencia de colegiación han sido puestos de relieve por la literatura científica sobre la profesión. Las instituciones reguladoras se han mostrado decisivas en su tarea de influir debido a su ascendiente sobre las identidades, con importantes consecuencias en términos de credibilidad y de posibilidades de progreso de las propias carreras de los profesionales (Scott, 2008; Barbour \& Lammers, 2015; Fernández-Esquinas, 2016) o en su capacidad para crear un marco cognitivo y reglamentario alrededor de las profesiones (Greenwood et al., 2002; Scott, 2008; Fernández Alcalde, 2010; Barbour \& Lammers, 2015; Alonso-Domínguez et al., 2016; Guilló \& Santiago, 2016).

En suma, se podría decir que ambas disciplinas comparten algunos problemas que han sido destacados de forma recurrente por la literatura sobre la profesión y que en el presente capítulo quedarán patentes en la sección dedicada a la perspectiva de los autores. Nos encontramos en un momento de devaluación, inestabilidad e incertidumbre profesional (Sigalat, 2016) de la sociología y la ciencia política en España que requiere ser más consciente de las dinámicas de los colegiados y estimular el sentimiento corporativo de sus profesionales (Fernández Alcalde, 2010, p. 12; Alonso-Domínguez et al., 2016; Guilló \& Santiago, 2016) a través del fortalecimiento de las funciones consultivas y ejecutivas de los colegios profesionales. Igualmente, a través de la capacidad de interpretación de la complejidad del mundo actual, que plantea retos, pero también oportunidades para que se pongan en valor y se utilicen las capacidades de los sociólogos 
y politólogos dispuestos a participar en equipos multidisciplinares (Domínguez-Amorós \& Gómez-Yáñez, 2016; FernándezEsquinas, 2016; Guilló \& Santiago, 2016; Sigalat, 2016).

Todo ello implica la revisión constante de los límites de las jurisdicciones (Greenwood et al., 2002), para lo que resultan determinantes las exigencias de los propios colegiados, pero también el surgimiento de nuevas necesidades que solo será posible de cubrir mediante la regulación estatal y autonómica de los colegios profesionales, como expertos sociales que son en el proceso de cambio de estas comunidades organizativas. La siguiente sección, dedicada a la regulación de los colegios profesionales en España, muestra, no obstante, que la legislación española solamente ha estimulado la colegiación cuando es obligatoria, socavando aún más el desarrollo profesional de disciplinas como la sociología o la ciencia política en las que no existe esa exigencia. También se verá cómo el fallido Anteproyecto de Ley de Servicios y Colegios Profesionales (ALSCP), que entre 2013 y 2015 estuvo presente en la agenda política española, pudo haber cambiado el devenir del conflicto entre la libertad de elección de la profesión y colegiación obligatoria.

\section{Profesiones reguladas, tituladas y de colegiación obligatoria}

La normativa sobre colegiación y ejercicio profesional en el mundo de la sociología y la ciencia política es la relativa a la legislación sobre la libre elección de profesión y la regulación de las profesiones tituladas, entendiendo por "profesiones reguladas" a aquellas actividades profesionales en las que existen una serie de requisitos para el acceso o el ejercicio, bien sea a través de determinadas cualificaciones profesionales o disposiciones legales, reglamentarias o administrativas (Real Decreto 1837/2008). Desde la entrada en vigor de la constitución española de 1978, el reconocimiento de los derechos y las libertades, entre los que se encuentran la libre elección profesional u oficio (artículo 35) y la 
regulación de las peculiaridades propias del régimen jurídico de los colegios profesionales y el ejercicio de las profesiones tituladas (artículo 36), exigen un conjunto de garantías en relación a los mismos, quedando recogidas, fundamentalmente, en el Capítulo IV de su Título Primero. Además, a estos dos derechos ubicados en la Sección $2^{a}$ Capítulo II del Título Primero, se les otorga un nivel de protección medio.

El artículo 35, que reconoce con carácter general el derecho a la libre elección de profesión u oficio, y el artículo 36, que establece la regulación de las profesiones tituladas, conforman la libertad de ejercicio profesional. Esta confluencia de preceptos nos lleva a una situación aparentemente paradójica. Por un lado, la libertad para elegir una determinada profesión es total, si bien su ejercicio se encuentra tutelado y, por ello, limitado, como en los casos en que la misma es amparada por un colegio profesional. En este sentido, la redacción actual del apartado 2 del artículo 3 de la Ley 2/1974 sobre colegios profesionales establece que "será requisito indispensable para el ejercicio de las profesiones hallarse incorporado al Colegio Profesional correspondiente cuando así lo establezca una ley estatal" (p. 4) y, como se apuntaba anteriormente, no pretende otra cosa que garantizar a la ciudadanía el ejercicio de la profesión titulada conforme al interés público, de acuerdo con algunas sentencias constitucionales (STC) como la STC 166/1992 o la STC 123/1978. El constituyente no ha establecido un modelo cerrado de colegio profesional, siempre que se respete el imperativo de una estructura interna y un funcionamiento democrático, de modo que el margen normativo del legislador es muy amplio a la hora de llevar a cabo la configuración de los colegios profesionales, permitiendo al legislador "optar por una configuración u otra de este tipo de entidades» (STC 3/2013, p. 26).

Por tanto, la naturaleza de los colegios profesionales goza de unas singularidades que separa a estos de otras formas organizativas. La principal es que son corporaciones de derecho público que ejercen funciones de naturaleza privada, por lo que podemos 
afirmar que se constituyen primordialmente para defender los fines privados de sus miembros, si bien también se encuentran entre sus objetivos algunos de interés público que van más allá de estos intereses particularistas. Este carácter mixto los configura como corporaciones cuya propia existencia y finalidad no depende exclusivamente de sus asociados, sino que también de los propios mandatos y órdenes del ordenamiento jurídico, al atribuirle funciones que parecen más propias de las administraciones públicas titulares de competencias propias (STC 76/1983, 23/1984 y 123/1987).

La Ley 2/1974 ha sufrido desde su entrada en vigor múltiples modificaciones. De todas ellas, la más significativa para los colegios profesionales se produce como consecuencia de la trasposición de la Directiva 2006/123/CE del Parlamento Europeo y del Consejo ${ }^{5}$, relativa a los servicios en el mercado interior, a través de la Ley 25/2009 de 22 de diciembre, más conocida como ley ómnibus, de modificación de diversas leyes para su adaptación a la ley sobre el libre acceso a las actividades de servicios y su ejercicio. A pesar de su importancia para la profesión, la ley ómnibus iba mucho más allá que la regulación de los colegios profesionales y pretendía, entre otros objetivos, "dinamizar en mayor medida el sector servicios y de alcanzar ganancias de competitividad en relación con nuestros socios europeos" (BOE, 2009, p. 108.510). La importancia de esta ley radica en que, con ella, se modifican casi medio centenar de leyes nacionales y, entre ellas, la Ley 2/1974, que era hasta ese momento la norma fundamental en la determinación del ejercicio de las funciones de los colegios en su ámbito territorial, las condiciones de acceso y el ejercicio profesional.

España forma parte de la Unión Europea compuesta por distintas instituciones supranacionales que poseen capacidad normativa. De entre las normas que emanan y obligan a los Estados miembros destacan las Directivas del Parlamento y del Consejo Europeo. 
Ahora bien, esta ley no modifica ni la naturaleza, ni la definición de los colegios profesionales en su redacción original, aunque sí sus fines. En la actualidad, a estas corporaciones les corresponde

la ordenación del ejercicio de las profesiones, la representación institucional exclusiva de las mismas cuando estén sujetas a colegiación obligatoria, la defensa de los intereses profesionales de los colegiados y la protección de los intereses de los consumidores y usuarios de los servicios de sus colegiados. (BOE, 2009, p. 108.517)

Desde el punto de vista de la representación institucional, esto resulta muy relevante, puesto que es exclusivo en las profesiones de colegiación obligatoria, lo que mantiene un doble rasero entre estas y aquellas para las que no existe ese imperativo, como la sociología y la ciencia política.

Las sucesivas reformas no abordaron el problema esencial del intrusismo profesional que afecta a sociólogos y politólogos, y quisieron ser superadas con el fallido Anteproyecto de Ley de Servicios y Colegios Profesionales (ALP), cuya tramitación se inició en 2013 y que fue retirado en 2015 por el gobierno del Partido Popular, sin oposición por parte del resto de formaciones políticas. Esta propuesta, abortada antes de nacer, no resolvía los problemas de injerencia en las profesiones desarrolladas por sociólogos y politólogos.

El controvertido texto preveía la eliminación (limitada) de la obligatoriedad de la colegiación en algunas profesiones reguladas, tratando de armonizar algunas medidas liberalizadoras con otras de marcado carácter intervencionista. El resultado final de este difícil equilibrio fue la oposición unánime de los colegios profesionales más poderosos y la retirada del anteproyecto a la espera de un contexto más favorable. El argumento principal para su paralización fue, no obstante, el inicio de conversaciones con la Comisión Europea sobre el acceso a las profesiones. 
En la regulación normativa, no parece tan importante lo que dice la ley como la posterior interpretación de los tribunales y, sobre todo, la exégesis del Tribunal Constitucional (TC) sobre todas estas cuestiones. La legislación anima a la colegiación, pero solo cuando es obligatoria y el poder y el estatus se consiguen si los colegios controlan la exigencia de colegiación. No existe ninguna alusión a las ocupaciones que resultan ignoradas en textos legislativos y en sentencias. En cuanto al conflicto entre libertad de elección de profesión y la colegiación obligatoria, la disputa ha sido resuelta por el TC para el que no existe tal contencioso, ya que entiende que la imposición obedece a razones estrictamente de interés público.

En todo caso, el artículo 3 del mencionado ALP (2013) no parece que hubiera tenido excesivas consecuencias para el desarrollo profesional de la sociología y la ciencia política, sino que, por el contrario, podría haber sido una fuente de oportunidades. El citado texto distinguía entre:

Profesión regulada: la actividad o conjunto de actividades profesionales, cuyo acceso, ejercicio o una de las modalidades de ejercicio estén condicionados de manera directa o indirecta, en virtud de disposiciones legales o reglamentarias, a la posesión de determinadas cualificaciones.

Profesión titulada: aquélla para cuyo acceso se exija estar en posesión de un título académico oficial de educación superior.

Profesión de colegiación obligatoria: aquella profesión titulada para cuyo ejercicio se exija la colegiación. (p. 8)

Esta tríada no suponía ni una verdadera libertad de colegiación ni una auténtica eliminación de su exigencia en el desarrollo de determinadas profesiones, entre las que se encuentran la sociología y la ciencia política. En la práctica, se pretendía reducir a la mitad las profesiones de colegiación obligatoria; se proyectaba mantener la obligatoriedad, entre otros, a médicos, dentistas, far- 
macéuticos, veterinarios, enfermeros, fisioterapeutas, podólogos, ópticos-optometristas, biólogos, físicos, químicos, geólogos, psicólogos, arquitectos, arquitectos técnicos, abogados, procuradores, graduados sociales, notarios, registradores de la propiedad y mercantiles, así como las ingenierías e ingenierías técnicas reguladas. Se dejaba fuera, entre otros, a los abogados de empresa, y los arquitectos e ingenieros en régimen de dependencia laboral que no firman proyectos ni dirigen obras, ni las ejecutan y, por supuesto, a los profesionales de los sectores objetos de este artículo. Esta separación de quienes firman proyectos o dirigen obras muestra, además, las dos vertientes de la sociología (extensible a la ciencia política) y la pugna y el conflicto entre quienes la ejercen como práctica laboral y los que la ven como crítica social (Blois, 2015), dificultando internamente un verdadero reconocimiento de estas ciencias como actividades profesionales y fomentando el desarrollo de actividades propias de otros profesionales ajenos a estos conocimientos.

En definitiva, más allá del fracasado intento de regulación, probablemente se haya perdido una oportunidad única para que el legislador no perciba exclusivamente a la sociología (y también a la ciencia política) como crítica social y reconozca su parte técnica. en tanto que "propone incrementar el grado de racionalidad de las instituciones y sociedades a partir del asesoramiento" (Blois, 2014, p. 116). Al mismo tiempo, se ha desaprovechado la ocasión de afrontar la colegiación obligatoria de profesionales de estos campos, evitando así reiterados e inadecuados desempeños de funciones propias de sociólogos y politólogos por titulados de otros ámbitos.

Por último, cabe decir que, a pesar del alto grado de descentralización política existente en España, el estado se reserva sus funciones y las Comunidades Autónomas tienen que respetarlas, algo que impide un exceso de dispersión normativa. El ALP (2013) pretendía, al mismo tiempo, extirpar cualquier posibilidad de autonomía a las Comunidades Autónomas, toda vez que única y exclusivamente una ley estatal podría crear obligaciones de 
colegiación, intentando evitar la obligatoriedad de colegiación de algunas profesiones en unas comunidades y en otras no. Esto, en última instancia, constituye un obstáculo para que la pretendida descentralización iniciada por los colegios profesionales de sociología y ciencia política tenga una efectividad (ver apartado 4) tan amplia como aparenta. No obstante, ni la discusión con relación a las eventuales consecuencias de la regulación, ni el propio proceso de descentralización se encuentran cerrados, como lo evidencian las respuestas de los representantes de las juntas de gobierno de los colegios profesionales españoles de sociología y ciencia política. En ese apartado empírico, cuya metodología se describe a continuación, los actores participantes refieren cómo se ha llevado a cabo la implantación de ambas disciplinas en el territorio.

\section{Metodología}

Ha quedado patente que el papel que desempeñan las asociaciones profesionales como mecanismos reguladores ha sido abundantemente teorizado, si bien resulta mucho más limitado su análisis empírico (Maijoor \& van Witteloostuijn, 1996; Greenwood et al., 2002). Es por ello que este artículo pretende abrir una vía de exploración alternativa a la discusión estratégica sobre las profesiones sociológica y politológica a partir de la elaboración de un cuestionario administrado a los Colegios de Sociología y Ciencia Política de España.

La metodología de investigación se diseñó con el objetivo de conocer 1) el volumen y perfil del cuerpo colegial y 2) su opinión ante algunos de los desafíos que afronta la profesión. Para ello, se levantó una muestra-universo comprendiendo todos los colegios profesionales de sociólogos y politólogos de España. A finales de 2018, cuando se llevó a cabo el trabajo de campo, solo se encontraban plenamente operativos los colegios de Madrid, Cataluña, Aragón, Castilla y León, Castilla La Mancha, Murcia, Andalucía y Asturias, y estaba recién constituido el de Canarias. 
Cabe reseñar que una comunidad como el País Vasco, de enorme relevancia cuantitativa y cualitativa, carece de Colegio, aunque mantiene una nutrida y activa asociación.

No existen colegios en La Rioja, Cantabria, Baleares, Ceuta y Melilla, de manera que la muestra comprende, finalmente, a los colegios constituidos y en pleno funcionamiento, más el Colegio Nacional, que recoge a los asociados de las regiones donde no se han constituido colegios profesionales.

Se utilizó un cuestionario mixto, con mayoría de preguntas cerradas y algunas abiertas que permitieran ampliar cuestiones $o$ matices que no se recogieran en las preguntas cerradas y se administró a través de la aplicación Surveymonkey. Todos los colegios respondieron de manera detallada al cuestionario, a excepción del catalán, que dejó numerosas preguntas sin responder, así que, cuando ha sido posible, se ha recopilado información desde su propia web.

Aunque el cuestionario recogía un número mucho más amplio de cuestiones sobre los colegios profesionales de sociología y ciencia política (ver anexo 1), en este trabajo presentamos los principales resultados de la encuesta acerca de los perfiles profesionales y las estrategias puestas en práctica por los colegios para mejorar su poder, influencia y, en consecuencia, su atractivo para los potenciales miembros. Las tablas se publican con base $\mathrm{N}=9$, puesto que las respuestas del colegio catalán no permiten incluirlo. En todo caso, cuando ha sido posible comprobar alguna de las respuestas en la web, se incluye una nota con un asterisco.

\section{Los colegios españoles de Sociología y Ciencia Política: perfiles y estrategias en el proceso de descentralización territorial}

De acuerdo con lo visto en el marco conceptual, la primera duda que nos asalta es qué se entiende por ser sociólogo en Espa- 
ña, un concepto que resulta, cuando menos, poco claro. No solo no significa lo mismo en unos países que en otros (Blois, 2015) sino que varía en función del objeto de estudio y el grado de institucionalización en cada territorio (Oliveira, 2019). En el caso de España, hablar de la institucionalización de las profesiones es hablar de colegios profesionales, tal y como se ha teorizado previamente. Es cierto que, en el caso de la sociología, existen también asociaciones, pero 1) carecen del perfil institucional que sí tienen los colegios, regulados por una ley específica y 2) suelen abrir sus estatutos tanto a sociólogos y politólogos como a personas cuya vida profesional esté relacionada con la sociología. En todo caso, hay un denominador común: se considera sociólogo a quien disponga de un título en sociología, sea de grado/licenciatura o de doctorado. Quedarían fuera de la definición los profesionales que ejerciéndola no están titulados como tales, incluyendo, sin embargo, a los titulados que no ejercen la profesión. Por último, como muestra de la indefinición del marco profesional en España, los colegios lo son de sociólogos y politólogos. La causa es que la licenciatura de sociología en España aparece en 1973 como carrera emparentada con la de ciencias políticas, con la que, hasta los años 90, compartía los tres primeros años de los cinco que tenía el plan de estudios. En la actualidad, pese a la mayor independencia de ambas disciplinas, se mantiene la colegiación conjunta.

\subsection{Una implantación débil y envejecida}

Con la excepción del Colegio de Madrid y del catalán, con unos 1.900 y 1.000 miembros, respectivamente, los colegios profesionales españoles presentan masas colegiales siempre inferiores a los doscientos profesionales, no llegando en la mayoría de los casos a los cien. Su reducido tamaño condiciona su desenvolvimiento $y$, en buena medida, el cumplimiento de sus objetivos corporativos. Son, además, colegios jóvenes; excepto el nacional, constituido en 1953, y el catalán, en 1989, todos datan de este siglo y principalmente de comienzos de su primera década. 
Además de esta juventud en cuanto a su creación y de su debilidad para incrementar la membresía, la información proporcionada por los colegios permite ofrecer algunas conclusiones sobre el perfil del sociólogo o politólogo colegiado. En primer lugar, destaca que predominan los colegiados talludos (ver tabla 1): solo el $24 \%$ los colegiados tienen menos de 35 años. La excepción son los colegios madrileño y andaluz, que declaran un $37 \%$ y $40 \%$ de jóvenes, respectivamente. Todo apunta a que los jóvenes sociólogos y politólogos no se colegian. Los motivos para explicar el relativo envejecimiento de los colegios son, desde la situación económica de los jóvenes graduados, precaria y poco propicia al pago de cuotas colegiales, hasta la puesta en cuestión de la necesidad de la existencia de los colegios, dudando de su representatividad y utilidad para los jóvenes egresados. Emergería la percepción de los colegios como organizaciones propias de profesionales establecidos, superadas por formas de organización y búsqueda de empleo alternativas, como las redes sociales $\mathrm{u}$ otras redes formales o informales.

Tabla 1:

\% del cuerpo colegial según grupos de edad y presupuesto

\begin{tabular}{|l|c|c|c|c|c|c|}
\hline Presupuesto & $22-34$ & $35-44$ & $45-54$ & +55 & Total & Media edad \\
\hline$+10.000 €$ & 24,5 & 26,7 & 21,5 & 25,5 & 100 & 44,1 \\
\hline $5.000-9.999$ & 23,8 & 26,2 & 31,3 & 16,2 & 100 & 46 \\
\hline $2.500-4.999$ & - & - & - & - & - & - \\
\hline-2.499 & 10 & 30 & 30 & 30 & 100 & 48 \\
\hline Media & 22,5 & 26,9 & 26,8 & 21,8 & 100 & 43,7 \\
\hline N & 9 & 9 & 9 & 9 & 9 & 9 \\
\hline
\end{tabular}

Fuente: Elaboración propia

Sin embargo, organizaciones, como las de Madrid y Andalucía que gestionan bolsas de empleo y prácticas potentes (ver tabla 2), aseguran que estas son un buen reclamo para la colegiación de los más jóvenes. Todo apuntaría a que la percepción de una función instrumental de los colegios incrementaría su base social 
joven. La duda es si ello es posible cuando las ofertas laborales son pocas y la influencia y capacidad de los colegios para gestionar bolsas de empleo escasas.

Tabla 2:

Servicios prestados por los colegios según presupuesto (\%)*

\begin{tabular}{|l|c|c|l|c|l|l|l|c|}
\hline $\begin{array}{l}\text { Presu- } \\
\text { puesto }\end{array}$ & $\begin{array}{l}\text { Bolsa de } \\
\text { empleo }\end{array}$ & $\begin{array}{l}\text { Prácticas } \\
\text { profesio- } \\
\text { nales }\end{array}$ & $\begin{array}{l}\text { Asesora- } \\
\text { miento } \\
\text { profe- } \\
\text { sional }\end{array}$ & $\begin{array}{l}\text { Servicios } \\
\text { legales }\end{array}$ & $\begin{array}{l}\text { Mutuas } \\
\text { sanita- } \\
\text { rias }\end{array}$ & $\begin{array}{l}\text { Networ- } \\
\text { king }\end{array}$ & $\begin{array}{l}\text { Arbitraje } \\
\text { profe- } \\
\text { sional }\end{array}$ & $\begin{array}{l}\text { Forma- } \\
\text { ción }\end{array}$ \\
\hline$+10.000 €$ & 75 & 100 & 100 & 75 & 50 & 75 & 25 & 100 \\
\hline $\begin{array}{l}5.000- \\
9.999\end{array}$ & 75 & 50 & 50 & 25 & 25 & 25 & 25 & 100 \\
\hline $\begin{array}{l}2.500- \\
4.999\end{array}$ & - & - & - & - & - & - & - & - \\
\hline-2.499 & 0 & 0 & 0 & 0 & 0 & 0 & 0 & 100 \\
\hline Media & 66,6 & 88,8 & 88,8 & 55,5 & 33,3 & 55,5 & 100 & 100 \\
\hline N & 9 & 9 & 9 & 9 & 9 & 9 & 9 & 9 \\
\hline
\end{tabular}

Fuente: Elaboración propia

*El Colegio catalán declara en su web ofrecer todos estos servicios

Se observa, además, que una amplia mayoría $(88,4 \%)$ del cuerpo colegial procede de licenciaturas o grados en ciencia política y sociología; un 5,4\% corresponde a titulados en sociología y ciencia política en segunda titulación, pero que ejercen la primera (médicos, economistas, ingenieros, etc.). Se pone de relieve también la disociación entre titulaciones y profesiones. Los titulados en sociología y ciencia política que ejercen como tales constituyen dos tercios de los colegiados. Entre ellos, es predominante el desempeño profesional en el sector público. El grupo más nutrido $(19,7 \%)$ se dedica a la docencia universitaria, seguido por un $18,6 \%$ que ejerce en el sector privado; un $17,9 \%$ en las administraciones públicas; y otro $9,4 \%$ en el tercer sector. El tercio restante-que suponemos infraestimado por ser menos proclive a la colegiación- desempeña oficios no relacionados con su título: un $19,5 \%$ lo hace en el sector privado, un 7,8\% en el sector público y otro $4,6 \%$ en el tercer sector, quedando un $10 \%$ de desempleados o en otras situaciones. 


\subsection{Hacia nuevas estrategias de los colegios profesionales}

Los colegios han adoptado estrategias para abrir camino a los titulados, mejorar su posición competitiva, superar las dificultades y hacer más visible la profesión. Dichas estrategias se plasman fundamentalmente en la prestación de servicios, pero también en la intención de aumentar el poder y la autonomía de las profesiones, recurriendo en este caso a convenios y acuerdos con empresas, administraciones públicas y organizaciones sindicales.

Las respuestas de los representantes de las juntas de gobierno de los colegios profesionales de sociólogos y politólogos permiten elaborar una tipología de los principales servicios prestados. Los convenios de prácticas con organizaciones empresariales, sindicales o administrativas son uno de los servicios más demandados y que más colegiados atrae, aunque los actores también refieren como actividades destacadas la prestación de servicios de asesoramiento profesional, los servicios legales o labores de networking, e incluso Madrid y el Colegio Nacional ofrecen la posibilidad de recibir atención sanitaria por parte de mutuas. Las actividades formativas constituyen, en algunos casos, fuentes alternativas de financiación. El madrileño llega a ofrecer un Máster en Recursos Humanos y una Escuela de Verano, el catalán ofrece otro de Técnicas de Investigación, así como una amplia gama de cursos, subvencionados o de formación continua.

También son objeto de interés la puesta en marcha de iniciativas y actividades con el objetivo de dar visibilidad a la profesión, al tiempo que se ofrece un servicio a la comunidad: publicaciones, congresos, foros u observatorios que, en menor o mayor medida, desarrollan todos los colegios. Todos disponen de página web y cuentas en Twitter y Facebook, sin embargo, no todos cumplen con el requisito legal de disponer de ventanilla electrónica que permita realizar algunas gestiones como altas y bajas en línea o reclamaciones, ni siquiera mediante el envío de un correo electrónico (ver tabla 3). 
Tabla 3:

Servicios web y redes sociales según presupuesto (\%)*

\begin{tabular}{|l|c|c|c|c|c|c|}
\hline $\begin{array}{l}\text { Presu- } \\
\text { puesto }\end{array}$ & Web & $\begin{array}{l}\text { Ventanilla } \\
\text { en línea }\end{array}$ & \multicolumn{1}{l|}{ Blog } & Twitter & Facebook & Instagram \\
\hline$+10.000 €$ & 100 & 100 & 25 & 100 & 100 & 25 \\
\hline $\begin{array}{l}5.000- \\
9.999\end{array}$ & 100 & 25 & 50 & 100 & 100 & 0 \\
\hline $\begin{array}{l}2.500- \\
4.999\end{array}$ & - & - & - & - & - & - \\
\hline-2.499 & 100 & 100 & 0 & 100 & 100 & 0 \\
\hline Media & 100 & 77,7 & 33,3 & 100 & 100 & 11,1 \\
\hline N & 9 & 9 & 9 & 9 & 9 & 9 \\
\hline
\end{tabular}

Fuente: Elaboración propia

*El Colegio catalán declara en su web ofrecer todos estos servicios

La mayoría de los colegios tratan igualmente de cumplir con su función de buscar autonomía y poder para las profesiones (ver tabla 4). Así, muchos de ellos han firmado convenios con administraciones públicas y empresas, y reciben contratos de consultoría y asesoramiento, incluyendo proyectos financiados por la Unión Europea. Una buena parte recibe encargos de asesoramiento o peritaje y todos los colegios, excepto uno, han participado o participan en órganos consultivos o asesores y han formado parte de mesas de contratación o en tribunales para selección de puestos de trabajo para las administraciones públicas. Finalmente, todas las corporaciones, salvo una, editan publicaciones o gestionan observatorios que les sirven de altavoz profesional y les proporciona cierta notoriedad social. 
Tabla 4:

Influencia social (varios parámetros) según presupuesto (\%)

\begin{tabular}{|l|l|l|l|c|l|l|l|}
\hline $\begin{array}{l}\text { Presu- } \\
\text { puesto }\end{array}$ & $\begin{array}{l}\text { Publi- } \\
\text { cación, } \\
\text { observa- } \\
\text { torios }\end{array}$ & $\begin{array}{l}\text { Consul- } \\
\text { toría }\end{array}$ & $\begin{array}{l}\text { Forma } \\
\text { de con- } \\
\text { venios }\end{array}$ & Peritajes & $\begin{array}{l}\text { Arbi- } \\
\text { traje }\end{array}$ & $\begin{array}{l}\text { Partici- } \\
\text { pación } \\
\text { comités, } \\
\text { conse- } \\
\text { jos,.. }\end{array}$ & $\begin{array}{l}\text { Con- } \\
\text { cursos, } \\
\text { meas, } \\
\text { tribuna- } \\
\text { les. }\end{array}$ \\
\hline$+10.000 €$ & 100 & 75 & 100 & 75 & 25 & 75 & 75 \\
\hline $\begin{array}{l}5.000- \\
9.999\end{array}$ & 50 & 100 & 50 & 25 & 25 & 100 & 100 \\
\hline $\begin{array}{l}2.500- \\
4.999\end{array}$ & - & - & - & - & - & - & \\
\hline-2.499 & 100 & 0 & 0 & 0 & 0 & 100 & 100 \\
\hline Media & 77,7 & 77,7 & 66,6 & 55,5 & 22,2 & 88,8 & 88,8 \\
\hline N & 9 & 9 & 9 & 9 & 9 & 9 & 9 \\
\hline
\end{tabular}

Fuente: Elaboración propia

Las respuestas de los representantes colegiales revelan dilemas estratégicos que atañen al ser de los colegios y de la propia profesión. En cuanto a la descentralización de los colegios (ver tabla 5), una mitad opina que ha sido buena, permitiendo adaptarse mejor al territorio. La otra plantea dudas sobre la capacidad para prestar las funciones que la ley atribuye a los colegios profesionales, pero sí se observa un acuerdo en la deficiente comunicación entre colegios y se intuye, de manera latente, que no hay coordinación de acciones y planteamientos comunes a nivel nacional. De ahí que todas las organizaciones crean que es necesario mejorar la conexión entre ellas, proponiendo diversos mecanismos: desde compartir bases de datos hasta llevar actuaciones coordinadas en defensa de la profesión, pasando por establecer algún tipo de servicios médicos o jurídicos inviables a escala regional pero no en la nacional. 
Tabla 5:

Puntos de vista favorables sobre distintos aspectos estratégicos

\begin{tabular}{|l|c|c|c|}
\hline Presupuesto & $\begin{array}{c}\text { ¿Cree que la } \\
\text { descentralización } \\
\text { ha mejorado la } \\
\text { actividad de los } \\
\text { colegios? }\end{array}$ & $\begin{array}{c}\text { Cree necesaria una } \\
\text { mayor } \\
\text { coordinación inter- } \\
\text { colegial }\end{array}$ & $\begin{array}{c}\text { Cree que los } \\
\text { colegios deberían } \\
\text { abrirse a otros } \\
\text { grados }\end{array}$ \\
\hline$+10.000 €$ & 50 & 100 & 100 \\
\hline $5.000-9.999$ & 75 & 100 & 25 \\
\hline $2.500-4.999$ & 100 & 100 & $0^{*}$ \\
\hline-2.499 & 66,6 & 100 & 55,5 \\
\hline Media & 9 & 9 & 9 \\
\hline $\mathrm{N}$ & & & \\
\hline
\end{tabular}

Fuente: Elaboración propia

*Expresan dudas

En cuanto a los impactos (positivos o negativos) de una posible ley de liberalización de servicios profesionales, como la que proponía el fallido anteproyecto de ley, la opinión más extendida es que, al no ser de colegiación obligatoria, serán escasos, mientras que el asunto referido a qué profesionales deben acoger los colegios y si esto afecta a su esencia resulta algo controvertido y con numerosas aristas (ver gráficos 1 y 2). Hay una mayoría favorable, un $80 \%$, si bien con dudas, a abrir los colegios a profesionales de la sociología y la ciencia política no titulados como tales, favoreciendo profesiones y no titulaciones.

También surge la posibilidad de abrirse a profesiones y titulaciones afines y, por lo general, más numerosas. Sin embargo, las razones que justifican esta posición parecen más prácticas (ganar tamaño y, por tanto, músculo en influencia) que de otro tipo. Aparece aquí un problema estratégico: si se trata de ganar volumen que haga a los colegios más competitivos, la pregunta es si será acertado abrirlos a otras titulaciones o a profesionales que ejercen como sociólogos o politólogos, pero la paradoja de que la mayor visibilidad de los colegios suponga la invisibilización de los titulados en sociología y ciencia política levanta sombras de duda. 
Gráfico 1

¿Por qué cree que los colegios no deben abrirse a otros grados?

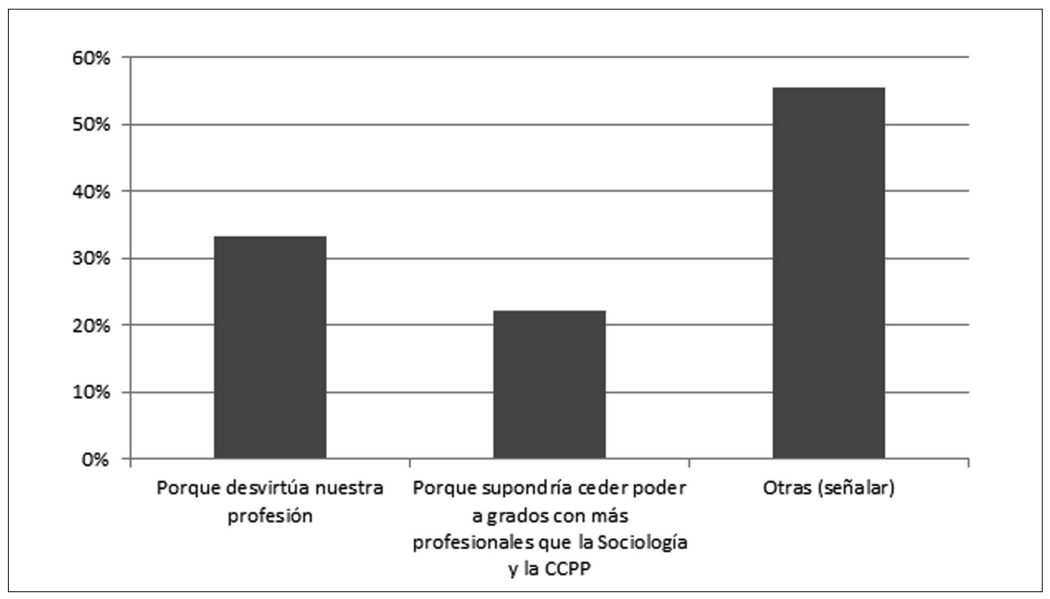

Fuente: Elaboración propia

\section{Gráfico 2}

¿Por qué cree que los colegios sí deben abrirse a otros grados?

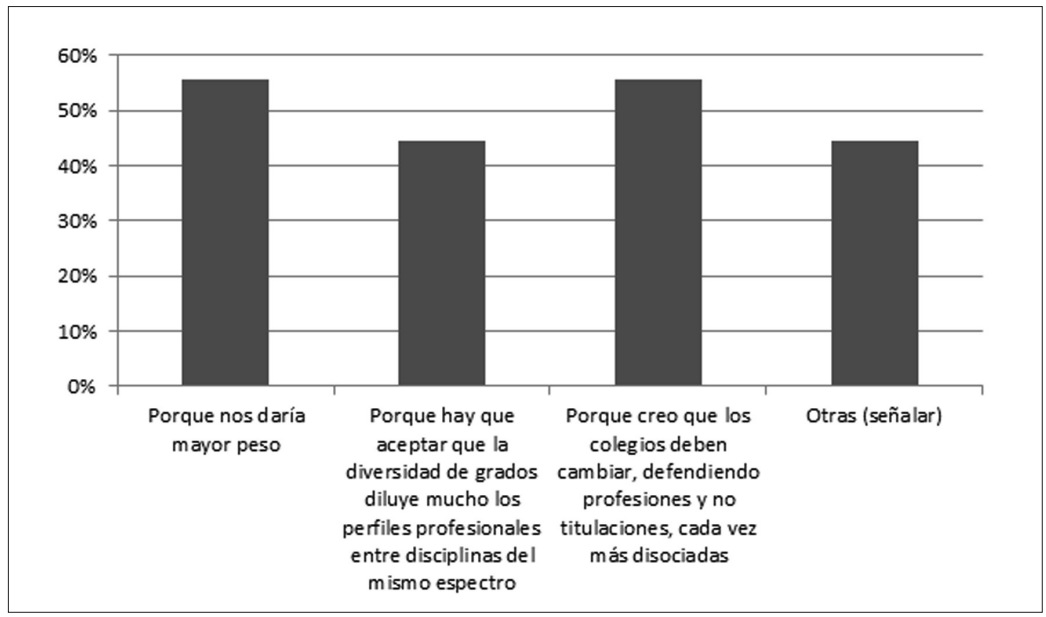

Fuente: Elaboración propia

Finalmente, surge implícita una cuestión clave: definir qué es un sociólogo o un politólogo, una cuestión que no está nada clara, como han subrayado recientemente autores como Martín Aranaga (2019) en España, o Blois $(2014,2015)$ en Latinoamérica. Por 
ejemplo, la eclosión de las nuevas tecnologías de la información y su creciente aplicación en la investigación social han abierto las puertas de la profesión a personas procedentes de la informática o ingenierías con cierta intuición sociológica o formación complementaria, desafiando las convenciones tradicionales sobre la profesión de sociólogo o politólogo.

En el siguiente apartado se discuten estas y otras cuestiones en torno a la profesión del sociólogo y el politólogo que se han revelado importantes en la presente investigación, tanto a nivel conceptual como práctico.

\section{Discusión y conclusiones}

La revisión de la literatura ha puesto de relieve que los colegios profesionales de sociólogos y politólogos presentan dificultades para cumplir no ya con los objetivos que les marca la ley de 1974, sino para alcanzar otros latentes en el mercado profesional y en la mejora de posiciones en la disputa por el espacio social de acción y de influencia profesional, al menos en las regiones donde la estructura colegial es más débil. Tampoco parece claro que se alcancen objetivos, como reforzar el sentido de pertenencia, los vínculos intragrupales y otros aspectos menos perceptibles, como las convicciones, valores y creencias relacionadas con la profesión, configurando un escenario donde ambos incumplimientos se retroalimentan.

Desde este planteamiento teórico, la investigación ha contribuido a caracterizar una competencia cada vez más difusa como consecuencia tanto de la aparición de nuevas titulaciones concomitantes o que se solapan con la sociología y la ciencia política, como por la inevitable competencia profesional que llega desde otras titulaciones, más aún, cuando el rol profesional del sociólogo, sobre todo fuera de la academia, está tan poco definido.

La descentralización que ha recorrido la mayor parte de los colegios profesionales tampoco ayuda. El análisis empírico rea- 
lizado ha puesto de manifiesto que los colegios que han nacido y se han consolidado al amparo de esta segregación son, por lo general, y con la excepción del madrileño y del catalán, pequeños y débiles, manejan escasos presupuestos, apenas poseen estructuras orgánicas y ofrecen pocos servicios, muchas veces dependiendo de la voluntad de los colegiados, así que resultan poco atractivos para los jóvenes titulados, presentando una demografía envejecida y una actividad que depende más de la disponibilidad y entusiasmo de su cuerpo colegial que de estructuras consolidadas. El artículo ha encontrado, igualmente, una sólida relación entre este proceso y el tamaño, la variable que define casi todo en el universo colegial de la sociología y la politología española, desde la carta de servicios ofrecida hasta las actitudes ante la liberalización profesional.

Por todo ello, la reflexión sobre el futuro de los colegios está llena de dudas y controversias. Las actitudes ante el proceso descentralizador y sobre las potenciales oportunidades derivadas de la aplazada trasposición de la Directiva Europea de Liberalización de Servicios Profesionales, son ambivalentes. Además de no verse clara la estrategia o su oportunidad, surgen vacilaciones sobre si la mayor visibilidad que ofrecerían a los colegios no sería en detrimento de la profesión. Hay otro escenario, apenas contemplado, que es el inverso: asociar nuestras disciplinas con otros colegios, subsumiéndonos en ellos. Son cuestiones que nos llevan a preguntarnos, incluso, qué es un sociólogo o un politólogo.

Los dilemas y controversias están ahí y carecen de respuesta clara por parte de los colegios. En todo caso, es evidente que la situación colegial en España no es la mejor, especialmente de cara a competir con otras profesiones tradicionales o emergentes, pero más pujantes y, quizá, más definidas profesionalmente y con mayor espíritu corporativo. Por todo ello, se sugieren estrategias intermedias de coordinación entre colegios, que conduzcan a una mejor y más precisa definición profesional y su identidad, así como a la unificación de líneas de actuación hasta ahora no suficientemente exploradas para ganar peso social, operatividad y 
capacidad para cumplir con sus funciones legales, soslayando algunas de las amenazas que penden sobre la profesión y los propios colegios profesionales.

No parece acertado finalizar sin resaltar algunas de las limitaciones del presente artículo. La principal es de carácter metodológico, derivada de la utilización de un cuestionario compuesto principalmente por preguntas cerradas, una circunstancia que, ciertamente, resta capacidad informativa y de discusión al texto. Pero la ambición de este trabajo era meramente exploratoria, casi de inventario, por lo que la investigación no se agota aquí, sino que pretende ser un punto de partida para abordar un diagnóstico más en profundidad, que analice la viabilidad de las estrategias para la mejora de posiciones profesionales y el desarrollo de una identidad profesional a través de los resultados alcanzados ante los agentes de empleo, del análisis de redes sociales y, desde luego, buscando variables explicativas al éxito o fracaso de esas estrategias.

\section{Referencias}

Abbott, A. (1988). The system of professions: An essay on the division of expert labor. Chicago: The University of Chicago Press. Disponible en: https://doi.org/10.7208/chicago/9780226189666.001.0001

Almond, G. (1999). Mesas separadas: escuelas y corrientes en las ciencias políticas. En G. Almond (Ed.), Una disciplina segmentada. Escuelas y corrientes en las ciencias políticas (pp. 39-62). México D.F.: Fondo de Cultura Económica.

Alonso-Domínguez, Á., Eguren, N. \& González Fernández, A. (2016). La sociología como profesión: Estrategias de potenciación de la profesión sociológica en el Principado de Asturias. Revista Española de Sociología, 25(3), 171-188. Disponible en: http:/ / dx.doi.org/10.22325/fes/res.2016.13

Anteproyecto de Ley de Servicios y Colegios Profesionales (2013). Gobierno de España, Ministerio de Economía y Empresa. Disponible en: http://www.mineco.gob.es/stfls/mineco/ prensa/ficheros/noticias/2013/130802_APLSCP_2_agosto.pdf 
Ashforth, B., Rogers, K. \& Corley, K. (2011). Identity in organizations: exploring cross-level dynamics. Organization Science, 22(5), 1144-1156. Disponible en: https://doi.org/10.1287/ orsc. 1100.0591

Barbour, J. \& Lammers, J. (2015). Measuring professional identity: a review of the literature and a multilevel confirmatory factor analysis of professional identity constructs. Journal of Professions and Organization, 2(1), 38-60. Disponible en: https:// doi.org/10.1093/jpo/jou009

Blois, J. P. (2014). Mercado, opinión pública y sociología en Argentina. Nómadas (Col), (41), 115-129.

Blois, J. P. (2015). La institucionalización y profesionalización de la sociología en Brasil y Argentina. Formación, organización e intervención de los sociólogos. Estudios Sociológicos, 33(99), 633-658. Disponible en: https://doi.org/10.24201/ es.2015v33n99.1393

Blois, J. P. (2017). La sociología como profesión en Argentina desde mediados del siglo XX. Cadernos de Pesquisa, 47(165), 938-962. Disponible en: https:/ / doi.org/10.1590/198053144638

Bolívar, A., Gallego, M. J., León, M. J. \& Pérez, P. (2005). Políticas educativas de reforma e identidades profesionales: el caso de la educación secundaria en España. Archivos Analíticos de Políticas Educativas, 13(45), 1-51. Disponible en: https:/ / digibug.ugr.es/bitstream/handle/10481/53068/artículo_ redalyc $\_275020513045$.pdf? sequence $=1 \&$ isAllowed $=\mathrm{y}$

Bourdieu, P. (2002). Campo de poder, campo intelectual. Buenos Aires: Montressor Jungla Simbólica.

Brint, S. (1990). Rethinking the policy influence of experts: From general characterizations to analysis of variation. Sociological Forum, 5(3), 361-385. Disponible en: https:/ / doi.org/10.1007/ BF01115092

Bulcourf, P. \& Vázquez, J. C. (2004). La ciencia política como profesión. PostData: Revista de Reflexión y Análisis Político, (10), 255-304.

Constitución española. (1978). Disponible en: http://www.congreso. es/consti/

Cotarelo, R. (1994). Ciencia política y de la administración. En R. Reyes (Ed.), Las ciencias sociales en España: Historia inmediata, crítica y perspectivas. Madrid: Editorial Complutense. 
Domínguez-Amorós, M. \& Gómez-Yáñez, J. A. (2016). Formación y competencias en la profesión del sociólogo/a. Revista Española de Sociología, 25(3), 17-43. Disponible en: https:/ /doi. org/10.22325/fes/res.2016.6

Fernández-Esquinas, M. (2016). La profesión sociológica en el siglo XXI: Estrategias para potenciar la situación de la sociología en el mercado de trabajo. Revista Española de Sociología, 25(3), 213-224. Disponible en: http://dx.doi.org/10.22325/fes/ res.2016.16

Fernández Alcalde, J. B. (2010). La sociología no existe: Manifiesto o egiptización profesional. Intersticios. Revista Sociológica de Pensamiento Crítico, 4(2), 5-27. Disponible en: http:/ / www. intersticios.es/article/view/6240/4344.

Forni, P. y Nardone, M. (2007). ¿Cómo generar capital social en contextos de exclusión?: Experiencias de organizaciones comunitarias y sus redes sociales. Revista Temas Sociológicos, (12), 169-199.

Freidson, E. (1983). The theory of the professions: the state of the art. En R. Dingwall \& P. Lewis (Eds.). The sociology of the professions: Lawyers, Doctors and Others. Londres: Macmillan. Disponible en: https:/ / doi.org/10.1007/978-1-349-16979-5_2

Gildo de la Cruz, G. (2007). Alternativas en la complejidad de la estructura social: El caso de la conformación y apropiación de capital social en el Estado de Colima, México. Revista Temas Sociológicos, (12), 121-143. Disponible en: https:/ / doi. org/10.29344/07196458.12.220

Gómez-García, R. (2010). Variables en la profesionalización del trabajo social. Zerbitzuan, (48), 87-95.

Greenwood, R., Suddaby, R. \& Hinings, C. R. (2002). Theorizing change: The role of professional associations in the transformation of institutionalized fields. Academy of Management Journal, 45(1), 58-80. Disponible en: https://doi. org/10.5465/3069285

Guilló, C. \& Santiago, P. (2016). Asociacionismo en entidades profesionales y científicas de la sociología. Revista Española de Sociología, 25(3), 115-136. Disponible en: http://dx.doi. org/10.22325/fes/res.2016.10

Ley 2/1974 sobre Colegios Profesionales. Boletín Oficial del Estado, Madrid, España, 13 de febrero de 1974. Disponible en: https:/ / boe.es/buscar/act.php?id=BOE-A-1974-289. 
Ley 25/2009 de modificación de diversas leyes para su adaptación a la Ley sobre el libre acceso a las actividades de servicios y su ejercicio. Boletín Oficial del Estado, Madrid, España, 22 de diciembre de 2009. Disponible en: https:/ / www.boe.es / diario_boe/txt.php?id=BOE-A-2009-20725.

Machuca, A. (2008). La identidad profesional de los sociólogos. (Tesis de Maestría. México D. F.: Facultad Latinoamericana de Ciencias Sociales.

Maijoor, S. \& van Witteloostuijn, A. (1996). An empirical test of the resource-based theory: Strategic regulation in the Dutch audit industry. Strategic Management Journal, 17(7), 549569. Disponible en: https://doi.org/10.1002/(SICI)10970266(199607)17:7<549::AID-SMJ827>3.3.CO;2-I

Martín Aranaga, I. (2019). Las representaciones sociales sobre el oficio/profesión de sociólogo/a de las personas jóvenes tituladas en sociología en el País Vasco. Comunicación presentada en el XIII Congreso Español de Sociología, Valencia, España.

Nolin, J. (2008). In search of a new theory of professions. Science for the professions. Report No. 4. University of Borås. Disponible en: http://hb.diva-portal.org/smash/get/diva2:883755/ FULLTEXT01.pdf

Oliveira, A. (2019). O Ensino de Sociologia em Santa Fé (Argentina): Algumas Pistas Comparativas com o Caso Brasileiro. Revista TOMO, (34), 393-418. Disponible en: https://doi. org $/ 10.21669 /$ tomo.v0i34.9717

Real Decreto 1837/2008 por el que se incorporan al ordenamiento jurídico español la directiva 2005/36/CE, del Parlamento Europeo y del Consejo, de 7 de septiembre de 2005, y la directiva 2006/100/CE del Consejo, de 20 de noviembre de 2006, relativas al reconocimiento de cualificaciones profesionales, así como a determinados aspectos del ejercicio de la profesión de abogado. Disponible en: http://noticias.juridicas.com/base_datos/Admin/rd1837-2008.t1.html

Scott, W. R. (2008). Lords of the dance: Professionals as institutional agents. Organization Studies, 29(2), 219-238. Disponible en: https://doi.org/10.1177\%2F0170840607088151

Sigalat, E. (2016). La situación de los sociólogos españoles en el sector privado: Implicaciones para la práctica y el desarrollo profesional. Revista Española de Sociología, 25(3), 95-114. Disponible en: https:/ /doi.org/10.22325/fes/res.2016.9 
Torini, D. (2012). Formação e identidade profissional: a trajetória de egressos de Ciências Sociais (Tesis de Maestría). Sao Paulo: Universidade de São Paulo.

Wilensky, H. L. (1964). The professionalization of everyone? American Journal of Sociology, 70(2), 137-158. Disponible en: https:// doi.org/10.1086/223790 


\section{Anexo 1}

\section{Cuestionario}

Estamos preparando una publicación sobre la profesión de la sociología y la politología, que se destinará a obtener un perfil/ diagnóstico de nuestros colegios profesionales. Para recoger datos que permitan hacer ese análisis, le hacemos llegar este cuestionario. Sabemos que le va a ocupar un tiempo del que quizá apenas disponga. Pero creemos que entenderá perfectamente su interés y el de contestarlo con la mayor precisión y sinceridad posibles.

Dándole las gracias por el esfuerzo, reciba un cordial saludo

P1. Denominación de su Colegio

P2. ¿En qué año se constituyó su Colegio (indicar fecha de publicación de Estatutos en el Boletín correspondiente)?

P3. Marcar lo que corresponda:

1. En mi región hay al menos una facultad de sociología y/o ciencia política

2. En mi región hay un departamento universitario de sociología y/o ciencia política

3. En mi región hay un área universitaria de sociología y CCPP

4. No hay ninguna de las tres

Vamos a hacerle a hora unas preguntas sobre el perfil de sus colegiados. Si no sabe las cifras exactas, no dude en aportar unas aproximadas.

P4. ¿Cuántos colegiados tiene su Colegio? (señalar número) 
P5. ¿Qué titulaciones tienen los colegiados?

\begin{tabular}{|l|l|}
\hline Titulación & \% aproximado \\
\hline $\begin{array}{l}\text { Licenciados/graduado en sociología y/o políticas como } \\
\text { primera titulación }\end{array}$ & \\
\hline $\begin{array}{l}\text { Licenciado/graduados en disciplinas de ciencias y docto- } \\
\text { res en sociología/CCPP }\end{array}$ & \\
\hline $\begin{array}{l}\text { Licenciados/graduados en otras disciplinas humanísticas } \\
\text { o de letras y doctores en sociología/CCPP }\end{array}$ & \\
\hline $\begin{array}{l}\text { Titulados/graduados en otras disciplinas con la sociolo- } \\
\text { gía/CCPP como segunda carrera }\end{array}$ & \\
\hline Otras situaciones & \\
\hline
\end{tabular}

P6. ¿Qué perfiles profesionales tienen? Señale el \% aproximado en función de la actividad principal de los colegiados. Entre todas las etiquetas deben sumar el 100\%

\begin{tabular}{|l|l|}
\hline Situación & \% aproximado \\
\hline $\begin{array}{l}\text { Trabajadores en la empresa privada como sociólogos/po- } \\
\text { litólogos }\end{array}$ & \\
\hline $\begin{array}{l}\text { Trabajadores en la empresa privada no como sociólogos/ } \\
\text { politólogos }\end{array}$ & \\
\hline Trabajadores en las AAPP como sociólogos/politólogos & \\
\hline Trabajadores en las AAPP no como sociólogos/politólogos & \\
\hline Trabajadores en tercer sector como sociólogos/politólogos & \\
\hline $\begin{array}{l}\text { Trabajadores en tercer sector no como sociólogos/politó- } \\
\text { logos }\end{array}$ & \\
\hline PDI Universidad & \\
\hline Profesores de secundaria & \\
\hline Desempleados & \\
\hline Otras (señalar) & \\
\hline
\end{tabular}

P7. ¿Qué edades aproximadas tienen sus colegiados? Señale el \% aproximado. Entre todas las etiquetas deben sumar el 100\%

\begin{tabular}{|l|l|}
\hline Edad & $\%$ aproximado \\
\hline $22-35$ & \\
\hline $36-45$ & \\
\hline $46-55$ & \\
\hline Más de 55 & \\
\hline
\end{tabular}


P8. ¿Conocen colegas que pudiendo estar colegiados no lo están? Sí (pasa a pregunta 9)

No (pasa a pregunta 11)

NS

P9. ¿Y por qué cree que no lo están (respuesta múltiple)?

1. Porque no conocen el Colegio

2. Porque no les interesa colegiarse.

3. Porque creen que un Colegio no representa sus intereses

4. Por la percepción del Colegio como algo antiguo (corporativismo, institución...)

5. Por cuestiones económicas

6. Otras (señalar)

P10. ¿En qué ámbitos profesionales desempeñan su labor principal? (respuesta múltiple)

1. Trabajadores en la empresa privada como sociólogos/ politólogos

2. Trabajadores en la empresa privada no como sociólogos/politólogos

3. Trabajadores en las AAPP como sociólogos/politólogos

4. Trabajadores en las AAPP no como sociólogos/politólogos

5. Trabajadores en tercer sector como sociólogos/politólogos

6. Trabajadores en tercer sector no como sociólogos/politólogos

7. PDI Universidad

8. Profesores secundaria

9. Desempleados

10. Otras

Ahora nos gustaría que respondiera a unas preguntas sobre su colegio

P11. ¿Qué presupuesto anual tiene el colegio? (indicar rango)

1. De 0 a $2.500 €$

2. De 2.501 a $5.000 €$ 
3. De 5.001 a $10.000 €$

4. Más de $10,001 €$

P12. ¿Podría indicar el coste anual de la cuota de su colegio?

P13.¿Disponen de cuotas específicas (desempleo, discapacidad...)? Si las hubiera, señalar importe

P14.¿De dónde proceden sus ingresos? Señale el \% aproximado. Entre todas las etiquetas deben sumar el 100\%

\begin{tabular}{|l|l|}
\hline Concepto & $\%$ \\
\hline Cuotas de colegiados & \\
\hline Subvenciones y ayudas de las AAPP & \\
\hline Ayudas de empresas privadas & \\
\hline Ingresos por actividades profesionales & \\
\hline Ingresos por otras actividades (cursos congresos...) & \\
\hline Otras (señalar cuáles) & \\
\hline
\end{tabular}

P15. ¿Cuáles son sus principales capítulos de gasto? Señale el \% aproximado. Entre todas las etiquetas deben sumar el 100\%

\begin{tabular}{|l|l|}
\hline Concepto & $\%$ \\
\hline Salarios & \\
\hline Alquileres & \\
\hline Dietas/desplazamientos & \\
\hline Material & \\
\hline Actividades (señalar cuáles) & \\
\hline Otras (señalar cuáles) & \\
\hline
\end{tabular}

P16. Estructura de que disponen

1. Técnicos en plantilla (indicar número)

2. Personal administrativo (indicar número)

P17. Estructura de que disponen

1. Gestión web y RRSS externa

2. Gestión web y RRSS interna

3. Gestión económica externa

4. Gestión económica interna 
P18. Estructura de que disponen

Grupos de Trabajo (señalar cuáles)

P19. Estructura de que disponen

Otros (señalar cuáles)

P20. ¿Disponen de...?

1. Sede propia comprada

2. Sede propia alquilada

3. Sede cedida

4. Sede compartida con otros colegios, asociaciones...

5. Sede compartida con otros colegios, asociaciones... en instalaciones de terceros.

Ahora le vamos a hacer unas preguntas sobre los servicios profesionales ofrecidos a la comunidad

P21. Servicios ofrecidos a cada comunidad profesional.

\begin{tabular}{|l|l|l|l|l|}
\hline & Sí & No & Querríamos hacerlo & $\begin{array}{l}\text { ¿Por qué, si quieren, } \\
\text { no lo han hecho? }\end{array}$ \\
\hline Bolsa de empleo & & & & \\
\hline
\end{tabular}

P22. Servicios ofrecidos a cada comunidad profesional.

\begin{tabular}{|l|l|l|l|l|}
\hline & Sí & No & Querríamos hacerlo & $\begin{array}{l}\text { ¿Por qué, si quieren, } \\
\text { no lo han hecho? }\end{array}$ \\
\hline $\begin{array}{l}\text { Convenios y } \\
\text { prácticas con em- } \\
\text { presas y AAPP }\end{array}$ & & & & \\
\hline
\end{tabular}

P23. Servicios ofrecidos a cada comunidad profesional.

\begin{tabular}{|l|l|l|l|l|}
\hline & Sí & No & Querríamos hacerlo & $\begin{array}{l}\text { ¿Por qué, si quieren, } \\
\text { no lo han hecho? }\end{array}$ \\
\hline $\begin{array}{l}\text { Asesoramiento } \\
\text { profesional }\end{array}$ & & & & \\
\hline
\end{tabular}

P24. Servicios ofrecidos a cada comunidad profesional.

\begin{tabular}{|l|l|l|l|l|}
\hline & Sí & No & Querríamos hacerlo & $\begin{array}{l}\text { ¿Por qué, si quieren, } \\
\text { no lo han hecho? }\end{array}$ \\
\hline Servicios legales & & & & \\
\hline
\end{tabular}


P25. Servicios ofrecidos a cada comunidad profesional.

\begin{tabular}{|l|l|l|l|l|}
\hline & Sí & No & Querríamos hacerlo & $\begin{array}{l}\text { ¿Por qué, si quieren, } \\
\text { no lo han hecho? }\end{array}$ \\
\hline $\begin{array}{l}\text { Mutuas sanita- } \\
\text { rias, seguros }\end{array}$ & & & & \\
\hline
\end{tabular}

P26. Servicios ofrecidos a cada comunidad profesional.

\begin{tabular}{|l|l|l|l|l|}
\hline & Sí & No & Querríamos hacerlo & $\begin{array}{l}\text { ¿Por qué, si quieren, } \\
\text { no lo han hecho? }\end{array}$ \\
\hline $\begin{array}{l}\text { Networking } \\
\text { colegiados }\end{array}$ & & & & \\
\hline
\end{tabular}

P27. Servicios ofrecidos a cada comunidad profesional.

\begin{tabular}{|l|l|l|l|l|}
\hline & Sí & No & Querríamos hacerlo & $\begin{array}{l}\text { ¿Por qué, si quieren, } \\
\text { no lo han hecho? }\end{array}$ \\
\hline $\begin{array}{l}\text { Arbitraje profe- } \\
\text { sional }\end{array}$ & & & & \\
\hline
\end{tabular}

P28. Servicios ofrecidos a cada comunidad profesional.

\begin{tabular}{|l|l|l|l|l|}
\hline & Sí & No & Querríamos hacerlo & $\begin{array}{l}\text { ¿Por qué, si quieren, } \\
\text { no lo han hecho? }\end{array}$ \\
\hline $\begin{array}{l}\text { Formación seña- } \\
\text { lar (cuál) }\end{array}$ & & & & \\
\hline
\end{tabular}

P29. Servicios ofrecidos a cada comunidad profesional.

\begin{tabular}{|l|l|l|l|l|}
\hline & Sí & No & Querríamos hacerlo & $\begin{array}{l}\text { ¿Por qué, si quieren, } \\
\text { no lo han hecho? }\end{array}$ \\
\hline $\begin{array}{l}\text { Otros servicios } \\
\text { (señalar cuáles) }\end{array}$ & & & & \\
\hline
\end{tabular}

P30. ¿Dispone de página web?

Sí

No

P31. ¿La web permite, de acuerdo con la ley, la realización de trámites en línea (ventanilla única)?

Sí

No 
P32. Dispone su colegio de....

\begin{tabular}{|l|l|l|l|}
\hline & No & $\begin{array}{l}\text { Sí, se le da uso al } \\
\text { menos una vez a la } \\
\text { semana }\end{array}$ & $\begin{array}{l}\text { Sí, pero se le da un } \\
\text { uso esporádico }\end{array}$ \\
\hline Blog & & & \\
\hline Twitter & & & \\
\hline Facebook & & & \\
\hline Instagram & & & \\
\hline
\end{tabular}

P33. Posibilidad de interlocución con instituciones clave del entorno, tanto empresas como administraciones y tercer sector: obtención de puestos de trabajo, demandas de proyectos, financiación, divulgación, etc.

\begin{tabular}{|l|l|l|l|l|}
\hline $\begin{array}{l}\text { ¿Han firmado (o van a firmar) } \\
\text { emvenios de empleo con AAPP, } \\
\text { empresas, ONGs, etc? }\end{array}$ & Si & No & $\begin{array}{l}\text { Que- } \\
\text { rríamos } \\
\text { hacerlo }\end{array}$ & $\begin{array}{l}\text { ¿Por qué, } \\
\text { si quieren, } \\
\text { no lo han } \\
\text { hecho? }\end{array}$ \\
\hline $\begin{array}{l}\text { ¿Se solicitan trabajos profesionales } \\
\text { (consultoría) al Colegio? }\end{array}$ & & & & \\
\hline ¿Se solicitan peritajes, dictámenes, etc.? & & & & \\
\hline $\begin{array}{l}\text { ¿Llevan a cabo tareas de arbitraje } \\
\text { nacional o internacional? }\end{array}$ & & & & \\
\hline $\begin{array}{l}\text { ¿Se pide participación al Colegio en } \\
\text { comités consultivos, consejos aseso- } \\
\text { res, etc.? }\end{array}$ & & & & \\
\hline $\begin{array}{l}\text { ¿Forman parte sus colegiados, en ca- } \\
\text { lidad de tales en concursos públicos o } \\
\text { convocatorias de puestos de trabajo? }\end{array}$ & & & & \\
\hline $\begin{array}{l}\text { ¿Tienen alguna publicación, observa- } \\
\text { torio, plataforma de opinión...? }\end{array}$ & & & & \\
\hline Otras (señalar) & & & & \\
\hline
\end{tabular}


P34. ¿Cree que la descentralización autonómica ha contribuido a mejorar la labor de los Colegios?

Sí

No

Por qué

P35. ¿Cree que es necesaria una mayor coordinación entre colegios a través de un posible Consejo General de la Sociología y la CCPP (o similar)?

Sí

No

Por qué

P36. ¿Qué tareas cree que podrían coordinarse?

P37. ¿Cómo cree que puede afectar a su colegio una posible Ley de Servicios y Colegios profesionales que, de acuerdo con las directivas de la UE, liberalice ambos?

P38. ¿Cree que los colegios de sociología y CCPP deberían abrirse a otros grados?

No (pasa a 39)

Sí (pasa a 40)

Tengo dudas (Pasa a 41)

P39. ¿Por qué no? (respuesta múltiple)

1. Porque desvirtúa nuestra profesión

2. Porque supondría ceder poder a grados con más profesionales que la sociología y la CCPP

3. Otras (señalar)

P40. ¿Por qué sí?

1. Porque nos daría mayor peso.

2. Porque hay que aceptar que la diversidad de grados diluye mucho los perfiles profesionales entre disciplinas del mismo espectro.

3. Porque creo que los colegios deben cambiar, defendiendo profesiones y no titulaciones, cada vez más disociadas.

4. Otras (señalar) 
P41. Por último, le dejamos un espacio para que señale todo aquello que, a su juicio, querría decir y el cuestionario no le ha permitido expresar.

Muchas gracias por sus respuestas. 